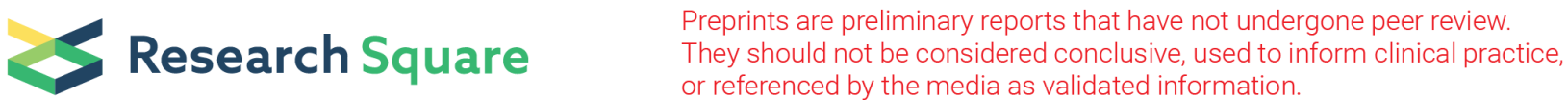

\section{Hepatic Macrophages Express Melanoma Differentiation-Associated Gene 5 in Nonalcoholic Steatohepatitis}

\section{SHOGO KAWAGUCHI ( $\sim$ kawaguchi.s@hirosaki-u.ac.jp )}

Hirosaki University Graduate School of Medicine https://orcid.org/0000-0001-5599-1110

\section{Hirotake Sakuraba}

Hirosaki University School of Medicine Graduate School of Medicine: Hirosaki Daigaku Igakubu Daigakuin Igaku Kenkyuka

\section{Momone Horiuchi}

Hirosaki University School of Medicine Graduate School of Medicine: Hirosaki Daigaku Igakubu Daigakuin Igaku Kenkyuka

\section{Jiangli Ding}

Hirosaki University School of Medicine Graduate School of Medicine: Hirosaki Daigaku Igakubu Daigakuin Igaku Kenkyuka

\section{Tomoh Matsumiya}

Hirosaki University School of Medicine Graduate School of Medicine: Hirosaki Daigaku Igakubu Daigakuin Igaku Kenkyuka

\section{Kazuhiko Seya}

Hirosaki University School of Medicine Graduate School of Medicine: Hirosaki Daigaku Igakubu Daigakuin Igaku Kenkyuka

\section{Chikara lino}

Hirosaki University School of Medicine Graduate School of Medicine: Hirosaki Daigaku Igakubu Daigakuin Igaku Kenkyuka

\section{Tetsu Endo}

Hirosaki University School of Medicine Graduate School of Medicine: Hirosaki Daigaku Igakubu Daigakuin Igaku Kenkyuka

\section{Hidezumi Kikuchi}

Hirosaki University School of Medicine Graduate School of Medicine: Hirosaki Daigaku Igakubu Daigakuin Igaku Kenkyuka

\section{Shukuko Yoshida}

Hirosaki University School of Medicine Graduate School of Medicine: Hirosaki Daigaku Igakubu Daigakuin Igaku Kenkyuka Hiroto Hiraga 
Hirosaki University School of Medicine Graduate School of Medicine: Hirosaki Daigaku Igakubu Daigakuin Igaku Kenkyuka

\section{Shinsaku Fukuda}

Hirosaki University School of Medicine Graduate School of Medicine: Hirosaki Daigaku Igakubu Daigakuin Igaku Kenkyuka

\section{Tadaatsu Imaizumi}

Hirosaki University School of Medicine Graduate School of Medicine: Hirosaki Daigaku Igakubu Daigakuin Igaku Kenkyuka

\section{Research Article}

Keywords: MDA5, NASH, macrophage, F4/80, CD11b, CDAHFD

Posted Date: May 7th, 2021

DOl: https://doi.org/10.21203/rs.3.rs-295080/v1

License: (c) (1) This work is licensed under a Creative Commons Attribution 4.0 International License. Read Full License

Version of Record: A version of this preprint was published at Inflammation on September 15th, 2021. See the published version at https://doi.org/10.1007/s10753-021-01550-8. 


\section{Abstract}

The activation of innate immune system is essential for the pathogenesis of nonalcoholic steatohepatitis (NASH). Among pattern recognition receptors, it is well-characterized that toll-like receptors (TLRs) are deeply involved in the development of NASH to reflect exposure of the liver to gut-driven endotoxins. In contrast, it has not been elucidated whether retinoic acid-inducible gene-I (RIG-I)-like receptors (RLRs) are similarly implicated in the disease progression. In the present study, we examined the expression of melanoma differentiation-associated antigen 5 (MDA5), known to be a member of RLRs, in a diet-induced murine model of NASH using western blotting and immunohistochemistry (IHC). The results of western blotting showed that hepatic expression of MDA5 was significantly increased 6 weeks after cholinedeficient L-amino acid-defined high-fat diet (CDAHFD). In IHC, MDA5-positive cells co-express F4/80 and CD11b, indicating they were activated macrophages, and these cells began to appear at 1 week after CDAHFD. Additionally, we performed IHC using liver biopsy specimens collected from eight patients with nonalcoholic fatty liver diseases (NAFLD), and found that MDA5 was expressed in CD11b-positive macrophages in six out of eight patients. The lobular inflammation score of NAFLD activity score tended to be higher in MDA5-positive cases than in MDA5-negative cases. Our findings suggest that MDA5 may be involved in the inflammation of NASH.

\section{Introduction}

Nonalcoholic fatty liver disease (NAFLD) is one of the most common liver diseases characterized by hepatic damage due to the excessive fat accumulation. NAFLD consists of nonalcoholic fatty liver (NAFL) and nonalcoholic steatohepatitis (NASH), and the latter is a more severe condition that can be a cause of liver cirrhosis and hepatocellular carcinoma (HCC). The multiple parallel hit theory is widely accepted as a mechanism of disease progression (1). Thus, hepatic inflammation appears at the same time or prior to steatosis, based on increased adipokines and gut dysbiosis. Adipokines such as tumor necrosis factor (TNF)-a, interleukin (IL)-6, and leptin released from hypertrophied adipocytes are involved not only in systemic microinflammation, but also in the development of insulin resistance. Additionally, intestinal barrier dysfunction and dysbiosis cause exposure of the liver to gut-driven endotoxins via the portal vein (2). These molecular mechanisms are thought to result in the onset and progression of NASH (3).

Hepatic macrophages are indispensable for the removal of exogenous antigens and activation of the immune system. They comprise of different subsets of cells, including resident macrophages, Kupffer cells (KCs), and monocyte-derived macrophages (MDM $\varphi$ ). KCs originate from yolk sac-derived progenitor cells, while $\operatorname{MDM} \varphi$ are derived from the bone marrow and differentiate from monocytes (4). KCs are characterized by $\mathrm{CD} 11 \mathrm{~b}^{\text {low }}, \mathrm{F} 4 / 80^{\text {high }}$ and $\mathrm{Clec} \mathrm{F}^{+}$, whereas $\mathrm{MDM} \varphi$ are characterized by $\mathrm{CD} 11 \mathrm{~b}^{+}$, $\mathrm{F} 4 / 80^{\text {int }}$, Ly $6 \mathrm{C}^{+}$and CSF1R ${ }^{+}$in mice (4). Following liver injury, $\mathrm{CD} 11 \mathrm{~b}^{+} \mathrm{F} 4 / 80^{+} \mathrm{MDM} \varphi$ are massively recruited into the liver (5). 
Pattern recognition receptors (PRRs) such as Toll-like receptors (TLRs) play a key role in the activation of macrophages. KCs promote TLR4-mediated TNF-a production in response to lipopolysaccharide (LPS) derived from the gut microbiota (6). Infiltrated macrophages are significantly decreased in the liver of TLR4-deficient mice (7). Therefore, TLR4 signaling is thought to be critical for NASH pathogenesis (6). In contrast, the involvement of retinoic acid-inducible gene-I (RIG-I)-like receptors (RLRs) in NASH remains unknown. Melanoma differentiation-associated gene 5 (MDA5) is a member of the DExH RNA helicase family of proteins as well as RIG-I, and functions as a cytoplasmic sensor of double-stranded RNA (8). MDA5 is also known to be an interferon (IFN)-stimulated gene (ISG) and has been implicated in chronic inflammation besides viral infection. We have previously reported that MDA5 is expressed in the gastric mucosa infected with Helicobacter pylori (9). Moreover, the increased expression of MDA5 was detected in the renal tissues in lupus nephritis and IgA nephropathy (10). Recently, growing evidence suggests an association of MDA5 with autoimmune diseases via upregulation of type I IFN signaling (11). These results indicate that MDA5 is implicated in a wide range of inflammation, including sterile inflammation. Although MDA5 may be associated with metabolic inflammation, including NASH, there have been no reports regarding the expression of MDA5 in NAFLD. Herein, we examined MDA5 expression in a dietinduced murine model of NASH and in eight patients with NAFLD, and found that MDA5 was expressed in activated macrophages in the liver of NASH patients.

\section{Materials And Methods}

\section{Materials}

A rabbit polyclonal anti-MDA5 antibody (\#29020) and a rabbit polyclonal anti-GAPDH antibody (\#2118) were purchased from Immuno-Biological Laboratories (Fujioka, Gunma, Japan) and Cell Signaling Technologies (Danvers, MA, USA), respectively. A rat polyclonal anti-F4/80 antibody (\#MCA497GA) and a rat polyclonal anti-CD11b antibody (\#GTX32495) were purchased from Bio-Rad Laboratories (Hercules, CA, USA) and GeneTex (Irvine, CA, USA), respectively. Alexa Fluor 488 anti-rabbit IgG antibody (A11008), Alexa Fluor 594 anti-rat IgG antibody (A21471), and 4'6'-diamidino-2-phenylindole (DAPI) were from Thermo Fisher Scientific (Rockford, IL, USA). Recombinant human TNF-a was purchased from Roche Diagnostics (Manheim, Germany). Lipopolysaccharide (LPS) from Escherichia coli was obtained from Sigma Aldrich (St. Louis, MO, USA). NucleoSpin RNA kit was purchased from Macherey-Nagel GmbH \& Co. KG (Düren, Germany). M-MLV reverse transcriptase and oligo(dT) 12-18 $_{18}$ were from Invitrogen (Federick, MD, USA). THUNDERBIRD Next SYBR qPCR mix was purchased from Toyobo (Osaka, Japan).

Polyvinylidene fluoride (PVDF) membranes and Luminata Crescendo Western HRP substrate were from Merck Millipore (Burlington, MA, USA).

\section{Animal Study}

Male C57BL/6J mice (8 weeks old) were obtained from CLEA Japan (Tokyo, Japan). All mice were maintained on a 12 -hours light/dark cycle at $22^{\circ} \mathrm{C}$ in a specific pathogen-free environment. After 1 week of acclimation, the mice were divided into a normal chow (NC) group and a choline-deficient, L-amino 
acid-defined high-fat diet (CDAHFD) (\#A06071302, Research Diets Inc., New Brunswick, NJ, USA) group (12). After 1 or 6 weeks, mice were sacrificed and livers were harvested for histological and biochemical analyses. All animal experiments were conducted in accordance with the Guidelines for Animal Experimentation of the Hirosaki University (Permit number: M20016).

\section{Cell Culture}

THP-1, a human monocytic leukemia cell line, was cultured in Roswell Park Memorial Institute (RPMI)1640 medium supplemented with Glutamax ${ }^{\mathrm{TM}}$ (Thermo Fisher Scientific, Waltham, MA, USA) containing $10 \%$ fetal bovine serum (FBS), $100 \mathrm{mg} / \mathrm{mL}$ penicillin, and $100 \mathrm{mg} / \mathrm{mL}$ streptomycin under $5 \%$ $\mathrm{CO}_{2}$ at $37^{\circ} \mathrm{C}$. To differentiate into macrophage-like cells, THP-1 cells were treated with $10 \mathrm{ng} / \mathrm{mL}$ of phorbol 12-myristate 13-acetate (PMA) for $48 \mathrm{~h}$. After another $24 \mathrm{~h}$ of incubation without PMA, the cells were stimulated with LPS or TNF-a for $24 \mathrm{~h}$.

\section{RNA Isolation and real-time quantitative PCR (qPCR)}

Total RNA was extracted from THP-1 cells and murine liver tissues using a NucleoSpin RNA kit according to the manufacturer's instructions, and cDNA was synthesized using the oligo(dT) ${ }_{12-18}$ primer and MMLV reverse transcriptase. A qPCR was performed using a Bio-Rad CFX real-time PCR thermocycler with THUNDERBIRD ${ }^{\text {TM }}$ Next SYBRß qPCR mix. The sequences of primers used are listed in Table 1. Each sample was run in triplicate.

\section{Western Blotting}

THP- 1 cells and murine liver tissues were lysed with RIPA buffer containing $0.2 \%$ proteinase inhibitors, and the lysates were centrifuged at $12,000 \mathrm{rpm}$ for $10 \mathrm{~min}$ at $4^{\circ} \mathrm{C}$. The supernatants were collected, and after determination of the protein concentration using BCA Protein Assay Reagent, equal amounts of the protein were loaded on a 10-20\% sodium dodecyl sulfate (SDS) polyacrylamide gel for electrophoresis. The separated proteins were transferred onto a PVDF membrane. After the membranes were blocked with Tris-buffered saline with Tween 20 (TBS-T, pH7.4) containing 5\% nonfat dry milk, they were incubated overnight at $4^{\circ} \mathrm{C}$ with a primary antibody against MDA5 (1:1000) or GAPDH (1:2000). The membranes were then incubated with an HRP-conjugated secondary antibody for $1 \mathrm{~h}$ at room temperature. Immunodetection was carried out using Luminata Crescendo substrate. The density of each band was measured using the ImageJ software (13).

\section{Histological Analysis}

Liver samples were obtained from eight patients (mean age $49.0 \pm 10.3$ ) who had undergone ultrasoundguided liver biopsy for the diagnosis of NASH at the Hirosaki University Hospital. The biopsy specimens were fixed in $10 \%$ formalin and embedded in paraffin. The sections were subjected to conventional hematoxylin and eosin (H\&E) staining and the NAFLD activity score (NAS) was evaluated based on Kleiner's criteria (14). The mean NAS of the eight patients was $4.4 \pm 0.2$ (steatosis $2.0 \pm 0$, lobular inflammation $1.5 \pm 0.2$, ballooning $0.9 \pm 0.1$ ). In addition, two patients who underwent liver biopsy for 
metastatic liver tumors without any existing liver diseases were included in this study, and the uninvolved area in the specimen was regarded as a normal control.

This study was approved by the Ethics Committee of the Hirosaki University Graduate School of Medicine (Permit number: 2018 - 152).

\section{Immunohistochemistry (IHC)}

After heat-induced antigen retrieval followed by blocking of endogenous peroxidase with $3 \%$ hydrogen peroxide, the tissue sections were blocked with $5 \%$ normal goat serum and $5 \%$ BSA in TBS-T for $1 \mathrm{~h}$. Sections were then incubated with a rabbit anti-MDA5 (1:250) and either a rat anti-F4/80 (1:250) or a rat anti-CD11b (1:250) antibodies overnight at $4^{\circ} \mathrm{C}$. After washing, the cells were stained with Alexa Fluor 488-conjugated anti-rabbit IgG antibody (1:250) and Alexa Fluor 594-congugated anti-rat IgG antibody (1:250) for $1 \mathrm{~h}$ at room temperature. After staining the nuclei with DAPI, the sections were visualized using confocal laser scanning microscopy (C1si; Nikon, Tokyo, Japan).

\section{Statistical Analysis}

All data are presented as the mean \pm SEM. Statistical differences were analyzed using Welch's $t$-test, oneway analysis of variance (ANOVA) or chi-squared test. Statistical significance was set at $p<0.05$.

\section{Results}

\section{Increased expression of MDA5 in the liver of CDAHFD-fed mice}

First, we analyzed MDA5 expression in the livers of CDAHFD-fed mice at 6 weeks. As shown in Fig. 1a, MDA5 expression was significantly higher in CDAHFD mice than in NC mice. In IHC, the immunoreactivity of MDA5 was not detected in the liver of NC mice, whereas patchy aggregation of MDA5-positive cells was detected in the liver of CDAHFD mice (Fig. 1b). Notably, we confirmed that MDA5-positive cells accumulated around the large lipid droplets (Fig. 1c). In the liver of NASH, the distinguishing histological feature is the presence of activated macrophages aggregated arround hepatocytes with fat degeneration known as "hepatic crown-like structure" (15). To investigate whether these MDA5-positive cells were macrophages or not, we performed double staining with antibodies against MDA5 and macrophage markers. As shown in Fig. 2, F4/80 and CD11b were co-expressed with MDA5, indicating that MDA5 was expressed in the activated macrophages.

Next, we examined whether MDA5 was induced in the early phase of NASH at 1 week. In western blotting, there was no difference in the expression of MDA5 between NC mice and CDAHFD mice (Fig. 3a), whereas in IHC, MDA5-positive cells began to appear even at 1 week after CDAHFD (Fig. 3b). We first confirmed that these cells were $\mathrm{CD} 11 \mathrm{~b}^{+}$(data not shown). qPCR results revealed that MDA5 mRNA was significantly upregulated at 1 week (Fig. 3c), whereas mRNA expression of proinflammatory cytokines, TNF-a, IL-6, and IL-12p40, remained almost unchanged (Fig. 3d, e, f). 


\section{Stimulation with LPS or TNF-a induces the expression of MDA5 in THP-1 cells}

We have previously reported that MDA5 expression is upregulated upon stimulation with LPS or TNF-a in some cell types $(16,17,18)$. To further elucidate the mechanisms mediating MDA5 expression in macrophages, we used cultured THP-1 cells. As shown in Fig. 4a and 4b, the expression of MDA5 mRNA was increased in THP-1 cells upon stimulation with either LPS or TNF- $a$ in a concentration-dependent manner. The expression of MDA5 mRNA reached the maximal level at $4 \mathrm{~h}$ after LPS stimulation, and then decreased gradually, whereas the expression was increased bimodally after TNF-a stimulation (Fig. 4c, d). Consistently, MDA5 protein level was augmented in a concentration-dependent manner with either stimulus (Fig. 4e). In contrast, stimulation with saturated fatty acid palmitate (C16:0) failed to induce MDA5 expression (Supplement. Figure 1a, b). As there was a possibility that some bioactive factors released from damaged hepatocytes may induce MDA5 expression in macrophages, we co-cultured THP1 with $\mathrm{HuH}-7$. However, stimulation of $\mathrm{HuH}-7$ with palmitate did not lead to the increased expression of MDA5 in THP-1 (Supplement. Figure 1c, d).

\section{Hepatic macrophages express MDA5 in human NAFLD}

Finally, we performed IHC with human liver tissues from eight patients with NAFLD and two control cases. Immunoreactivity of MDA5 was almost undetectable in the controls, whereas MDA5-positive cells were detected in six out of the eight cases with NAFLD (Fig. 5). These cells co-expressed MDA5 and $\mathrm{CD} 11 \mathrm{~b}$ as noted in the examinations in mice, suggesting that the infiltrating macrophages expressed MDA5 in human NAFLD also. As MDA5 expression was modest in two of the patients with NAFLD, these cases were considered as MDA5-negative cases, and we analyzed the histological differences between MDA5-positive and negative cases (Fig. 6a). There was no difference in NAS, steatosis score, and ballooning score between the two groups, whereas the lobular inflammation score tended to be higher in MDA5-positive cases than in MDA5-negative cases ( $p=0.103$, chi-squared test) (Fig. 6b, c, d, e). Cases with NAS $\geq 5$, who were considered as definite NASH, were all MDA5-positive cases (Fig. 6b).

\section{Discussion}

Hepatic macrophages play a key role in maintaining homeostasis and in the disease development in NAFLD (19). In the present study, we demonstrated that F4/80 CDAHFD-fed mice. $\mathrm{F} 4 / 80^{+}$macrophages are thought to be a heterogeneous cell population. $\mathrm{F} 4 / 80^{+}$ $\mathrm{CD} 11 \mathrm{~b}^{-} \mathrm{CD} 68^{+}$cells have potent phagocytic activity, whereas $\mathrm{F} 4 / 80^{+} \mathrm{CD} 11 \mathrm{~b}^{+} \mathrm{CD} 68^{+}$cells highly produce proinflammatory cytokines such as TNF-a and IL-12 in response to LPS (20). Interestingly, resident KCs undergo apoptosis during the NASH diet, and the loss of KCs is compensated by infiltrating MDM $\varphi(21)$. As $M D M \varphi$ are known to be $C D 11 b^{+}(4)$, it is thought that this subpopulation is infiltrated MDM $\varphi$ in NASH liver. $C D 11 b^{+}$macrophages are involved in high-fat diet-induced insulin resistance (22). Moreover, depletion of $\mathrm{CD} 11 \mathrm{~b}^{+}$macrophages by exposure to irradiation leads to marked inhibition of liver 
inflammation regardless of steatosis (23), suggesting that $\mathrm{CD} 11 \mathrm{~b}^{+}$macrophages promote the inflammation in NASH. Therefore, it is important to understand the pathogenesis of NASH to elucidate the precise functions of these subsets of cells. It should be noted that MDA5 was expressed in CD11 $\mathrm{b}^{+}$ macrophages and detected at 1 week after CDAHFD, that is, in the early phase of murine NASH. We confirmed that the area with steatosis was less than one-third of the liver on average, and that the mRNA expression of proinflammatory cytokines had not yet been upregulated at 1 week after CDAHFD. Although it has not been clarified when MDA5 is induced in the macrophages, MDA5 may have an influence on the immunological function of the cells.

MDA5-positive macrophages were detected in the livers of human NAFLD. The immunoreactivity of MDA5 was positive in more than $70 \%$ of human NAFLD cases, and the lobular inflammation score of NAS tended to be higher in MDA5-positive cases than in the negative cases, although the difference was not statistically significant. It can be speculated that the small sample size may have affected the statistical interpretations. However, there was no difference in the steatosis score, suggesting that whether MDA5 was expressed in the liver or not was determined by the magnitude of inflammation, not the severity of steatosis. In an in vitro study, we showed that LPS and TNF-a could upregulate the MDA5 expression, whereas saturated fatty acid palmitate failed to induce MDA5 in THP- 1 cells. These findings are consistent with the results obtained from the analysis of human samples as describe above. As MDA5 expression may be associated with the phase or degree of inflammation in human NAFLD, this molecule represents a potentially useful marker to differentiate NASH from NAFL.

The mechanism through which MDA5 modulates the inflammation remains to be elucidated. Activated macrophages secrete a variety of chemokines that infiltrate immune cells in inflamed sites. We have previously shown that LPS-induced C-X-C motif chemokine ligand 10 (CXCL10) production is significantly suppressed by knockdown of MDA5 in human mesangial cells and U373MG human astrocytoma cells $(16,17)$. Moreover, MDA5 knockdown resulted in a significant decrease in TNF-a-induced CXCL10 expression in HuH-7 and HLE human HCC cells (18). These results suggest that MDA5 may positively modulate TLR4 signaling and TNF-a-mediated immune responses. We speculate that MDA5-expressing $\operatorname{MDM} \varphi$ may exacerbate TLR4- or TNF-a-mediated inflammation in the liver via MDA5. The functional role of MDA5 in the pathogenesis of NASH should be further investigated in future studies.

\section{Conclusion}

In CDAHFD-fed mice, MDA5 was upregulated in the early phase of NASH. CD11 $\mathrm{b}^{+}$macrophages express MDA5 in the liver of human NAFLD patients. MDA5 may contribute not only to viral infection but also to metabolic inflammation in the liver.

\section{Declarations}

\section{Data Availability}


Not applicable.

\section{Funding}

This work was supported by JSPS KAKENHI Grant Number 20 K08346.

\section{Contributions}

S. Kawaguchi: Concenptualization, Resources, Investigation, Writing - original draft. H. Sakuraba: Concenptualization, Resources. M. Horiuchi, J. Ding, T. Matsumiya, and K. Seya: Investigation, Writing review \& editing. C. lino, and T. Endo: Investigation, Resources. H. Kikuchi: Concenptualization, Resources. S. Yoshida, and H. Hiraga: Methodology. S. Fukuda: Resources, Supervision. T. Imaizumi: Writing - review \& editing, Resources, Project administration.

\section{Ethics declarations}

\section{Ethics approval and Consent to participate}

All animal experiments were conducted in accordance with the Guidelines for Animal Experimentation of the Hirosaki University (Permit number: M20016). In retrospective studies using human liver biopsy specimens, informed consent was obtained from all patients in an opt-out format. This study protocol was approved by the Ethics Committee of the Hirosaki University Graduate School of Medicine (Permit number: 2018-152), and the study was performed in accordance with the 1964 declaration of Helsinki and its later amendments.

\section{Consent for publication}

Yes.

\section{Conflicts of interest}

The authors declare that they have no conflict of interest.

\section{References}

(1) Tilg, H., and A.R. Moschen. 2010. Evolution of inflammation in nonalcoholic fatty liver disease: the multiple parallel hits hypothesis. Hepatology. 52(5): 1836-1846.

(2) Chassaing, B., L. Etienne-Mesmin, and A.T. Gewirtz. 2014. Microbiota-liver axis in hepatic disease. Hepatology. 59(1): 328-339.

(3) Marra, F., and G. Svegliati-Baroni. 2018. Lipotoxicity and the gut-liver axis in NASH pathogenesis. $J$ Hepatol. 68(2): 280-295. 
(4) Wen, Y., J. Lambrecht, C. Ju, and F. Tacke. 2021. Hepatic macrophages in liver homeostasis and disease-diversity, plasticity and therapeutic opportunities. Cell Mol Immunol. 18(1): 45-56.

(5) Karlmark, K.R., R. Weiskirchen, H.W. Zimmermann, N. Gassler, F. Ginhoux, C. Weber, M. Merad, T. Luedde, C. Trautwein, and F. Tacke. 2009. Hepatic recruitment of the inflammatory Gr1+ monocyte subset upon liver injury promotes hepatic fibrosis. Hepatology. 50(1): 261-274.

(6) Rivera, CA., P. Adegboyega, N. van Rooijen, A. Tagalicud, M. Allman, and M. Wallace. 2007. Toll-like receptor-4 signaling and Kupffer cells play pivotal roles in the pathogenesis of non-alcoholic steatohepatitis. J Hepatol. 47(4): 571-579. \

(7) Miura, K., L. Yang, N. van Rooijen, H. Ohnishi, and E. Seki. 2012. Hepatic recruitment of macrophages promotes nonalcoholic steatohepatitis through CCR2. Am J Physiol Gastrointest Liver Physiol. 302(11): G1310-1321.

(8) Yoneyama, M., M. Kikuchi, K. Matsumoto, T. Imaizumi, M. Miyagishi, K. Taira, E. Foy, Y.M. Loo, M. Gale Jr., S. Akira, S. Yonehara, A. Kato, and T. Fujita. 2005. Shared and unique functions of the DExD/H-box helicases RIG-I, MDA5 and LGP2 in antiviral innate immunity. J Immunol. 175(5): 2851-2858.

(9) Tatsuta, T., T. Imaizumi, T. Shimoyama, M. Sawaya, T. Kunikazu, T. Matsumiya, K. Satoh, and S. Fukuda. 2012. Expression of melanoma differentiation associated gene 5 is increased in human gastric mucosa infected with Helicobacter pylori. J Clin Pathol. 65(9):839-843.

(10) Imaizumi, T., T. Aizawa-Yashiro, K. Tsuruga, H. Tanaka, T. Matsumiya, H. Yoshida, T. Tatsuta, F. Xing, R. Hayakari, and K. Satoh. 2012. Melanoma differentiation-associated gene 5 regulates the expression of a chemokine CXCL10 in human mesangial cells: implications for chronic inflammatory renal diseases. Tohoku J Exp Med. 228(1):17-26.

(11) Sadler, AJ. 2018. The role of MDA5 in the development of autoimmune disease. J Leukoc Biol. 103(2): 185-192.

(12) Matsumoto, M., N. Hada, Y. Sakamaki, A. Uno, T. Shiga, C. Tanaka, T. Ito, A. Katsume, and M. Sudoh. 2013. An improved mouse model that rapidly develops fibrosis in non-alcoholic steatohepatitis. Int J Exp Pathol. 94(2): 93-103.

(13) Schneider, CA., W.S. Rasband, and K.W. Eliceiri. 2012. NIH Image to ImageJ: 25 years of image analysis. Nature Methods. 9: 671-675.

(14) Kleiner, D.E., E.M. Brunt, M. Van Natta, C. Behling, M.J. Contos, O.W. Cummings, L.D. Ferrell, Y.C. Liu, M.S. Torbenson, A. Unalp-Arida, M. Yeh, A.J. McCullough, and A.J. Sanyal. For the Nonalcoholic Steatohepatitis Clinical Research Network. 2005. Design and validation of a histological scoring system for nonalcoholic fatty liver disease. Hepatology. 41(6): 1313-1321. 
(15) Itoh, M., H. Kato, T. Suganami, K. Konuma, Y. Marumoto, S. Terai, H. Sakugawa, S. Kanai, M. Hamaguchi, T. Fukaishi, S. Aoe, K. Akiyoshi, Y. Komohara, M. Takeya, I. Sakaida, and Y. Ogawa. 2013. Hepatic crown-like structure: a unique histological feature in non-alcoholic steatohepatitis in mice and humans. PLoS One. 8(12): e82163.

(16) Imaizumi, T., T. Aizawa-Yashiro, S. Watanabe, T. Matsumiya, H. Yoshida, T. Tatsuta, F. Xing, P. Meng, R. Hayakari, K. Tsuruga, and H. Tanaka. 2013. TLR4 signaling induces retinoic acid-inducible gene-I and melanoma differentiation-associated gene 5 in mesangial cells. J Nephrol. 26(5): 886-893.

(17) Imaizumi, T., K. Murakami, K. Ohta, H. Seki, T. Matsumiya, P. Meng, R. Hayakari, F. Xing, T. AizawaYashiro, T. Tatsuta, H. Yoshida, and H. Kijima. 2013. MDA5 and ISG56 mediate CXCL10 expression induced by toll-like receptor 4 activation in U373MG human astrocytoma cells. Neurosci Res. 76(4): 195206.

(18) Kawaguchi, S., H. Sakuraba, T. Haga, T. Matsumiya, K. Seya, T. Endo, N. Sawada, C. lino, H. Kikuchi, H. Hiraga, S. Fukuda, and T. Imaizumi. 2019. Melanoma differentiation-associated gene 5 positively modulates TNF-a-induced CXCL10 expression in cultured HuH-7 and HLE cells. Inflammation 42(6): 20952104.

(19) Li, H., Y. Zhou, H. Wang, M. Zhang, P. Qiu, M. Zhang, R. Zhang, Q. Zhao, and J. Liu. 2020. Crosstalk between liver macrophages and surrounding cells in nonalcoholic steatohepatitis. Front Immunol. 11: 1169.

(20) Kinoshita, M., T. Uchida, A. Sato, M. Nakashima, S. Shono, Y. Habu, H. Miyazaki, S. Hiroi, and S. Seki. 2010. Characterization of two F4/80-positive Kupffer cell subsets by their function and phenotype in mice. J Hepatol. 53(5):903-910.

(21) Seidman, J.S., T.D. Troutman, M. Sakai, A. Gola, N.J. Spann, H. Bennett, C.M. Bruni, Z. Ouyang, R.Z. Li, X. Sun, B.T. Vu, M.P. Pasillas, K.M. Ego, D. Gosselin, V.M. Link, L.W. Chong, R.M. Evans, B.M. Thompson, J.G. McDonald, M. Hosseini, J.L. Witztum, R.N. Gemain, and C.K. Glass. 2020. Niche-specific reprogramming of epigenetic landscapes derives myeloid cell diversity in nonalcoholic steatohepatitis. Immunity. 52(6): 1057-1074.e7.

(22) Zheng, C., Q. Yang, C. Xu, P. Shou, J. Cao, M. Jiang, Q. Chen, G. Cao, Y. Han, F. Li, W. Cao, L. Zhang, L. Zhang, Y. Shi, and Y. Wang. 2015. CD11b regulates obesity-induced insulin resistance via limiting alternative activation and proliferation of adipose tissue macrophages. Proc Natl Acad Sci U S A. 112(52): E7239-E7248.

(23) Nakashima, H., M. Nakashima, M. Kinoshita, M. Ikarashi, H. Miyazaki, H. Hanaka, J. Imaki, and S. Seki. 2016. Activation and increase of radio-sensitive CD11b+ recruited Kupffer cells / macrophages in diet-induced steatohepatitis in FGF5 deficient mice. Sci Rep. 6: 34466. 


\section{Tables}

Due to technical limitations, table 1 is only available as a download in the Supplemental Files section.

\section{Figures}

Fig. 1
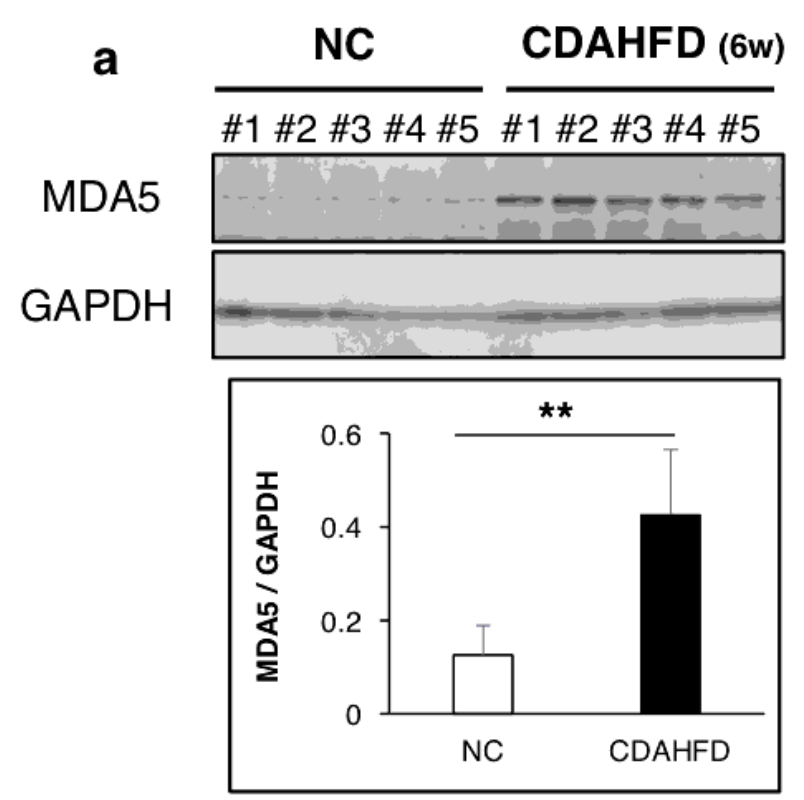

b
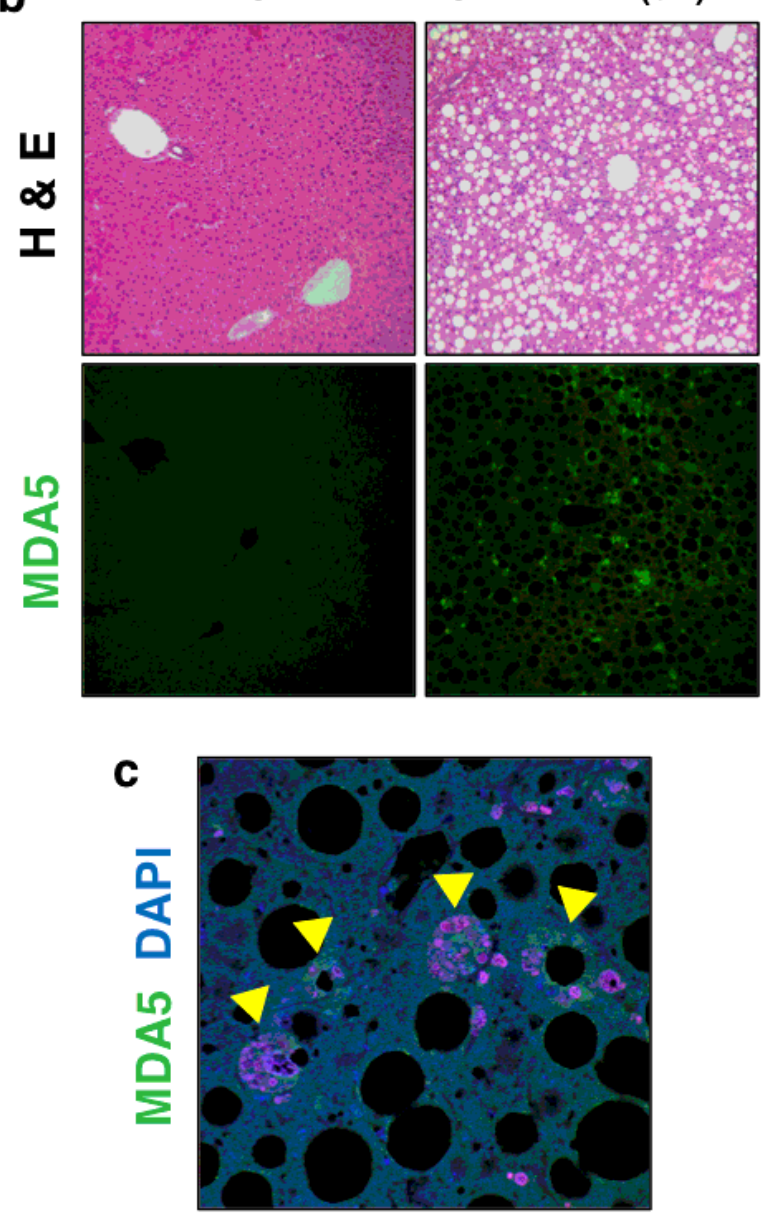

\section{Figure 1}

Expression of MDA5 in the liver of a diet-induced murine model of NASH. (a) Protein levels of MDA5 were significantly increased 6 weeks after choline-deficient L-amino acid-defined high-fat diet (CDAHFD). Results are presented as the mean \pm SEM of six mice in each group. ${ }^{* *} p<0.01$ vs. normal chow (NC)-fed mice. (b, c) Hematoxylin and eosin (H\&E) staining and immunohistochemical staining of MDA5 in the liver sections of NC and CDAHFD mice. Patchy accumulation of MDA5-positive cells was detected in CDAHFD mice. Original magnification: $x 100$. (c) Yellow arrowhead indicates MDA5-positive cells aggregated around large lipid droplets. Original magnification: $x 200$. 
Fig. 2
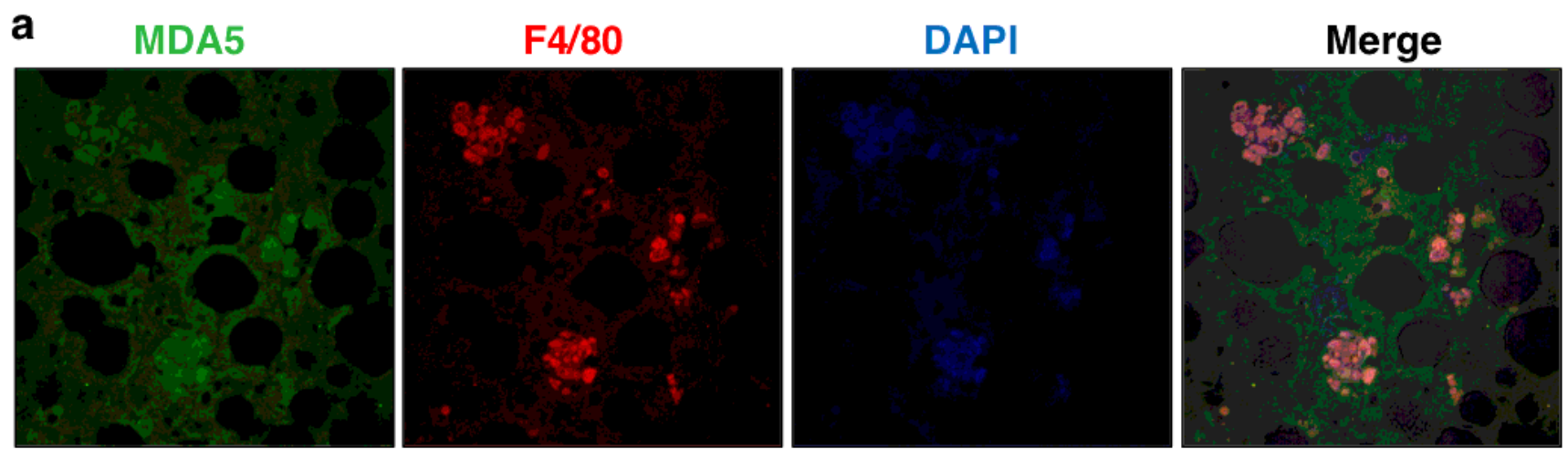

b

CD11b

DAPI

Merge
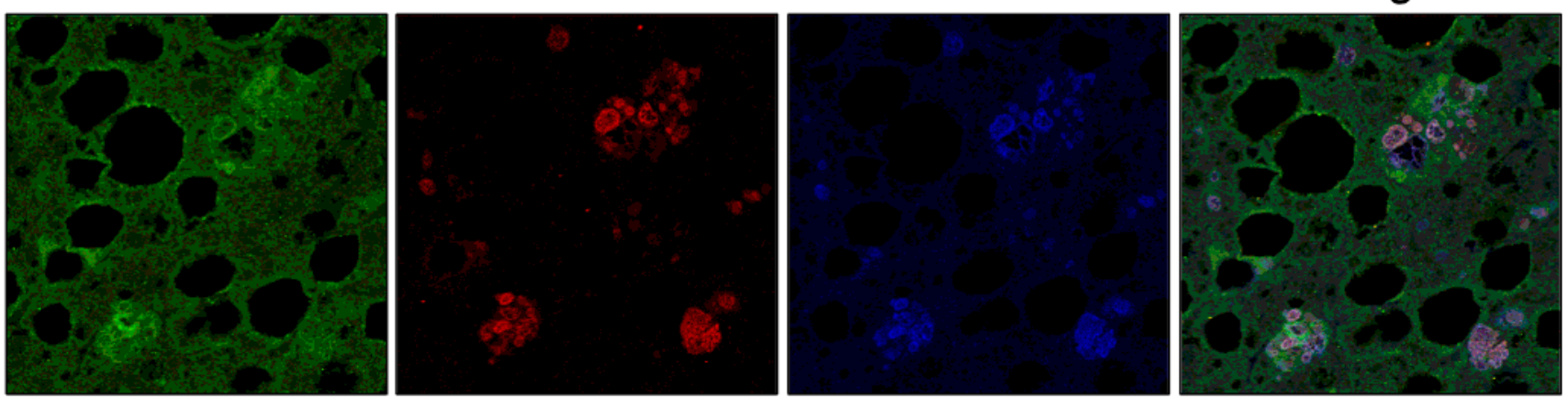

Figure 2

MDA5 is co-expressedwith F4/80 (a), or CD11b (b). Liver sections of CDAHFD mice were subjected to immunohistochemical staining for MDA5 (green), and either F4/80 (red) or CD11b (red). Cell nuclei were stained with DAPI (blue). Representative images are shown. Original magnification: x200. 
Fig. 3
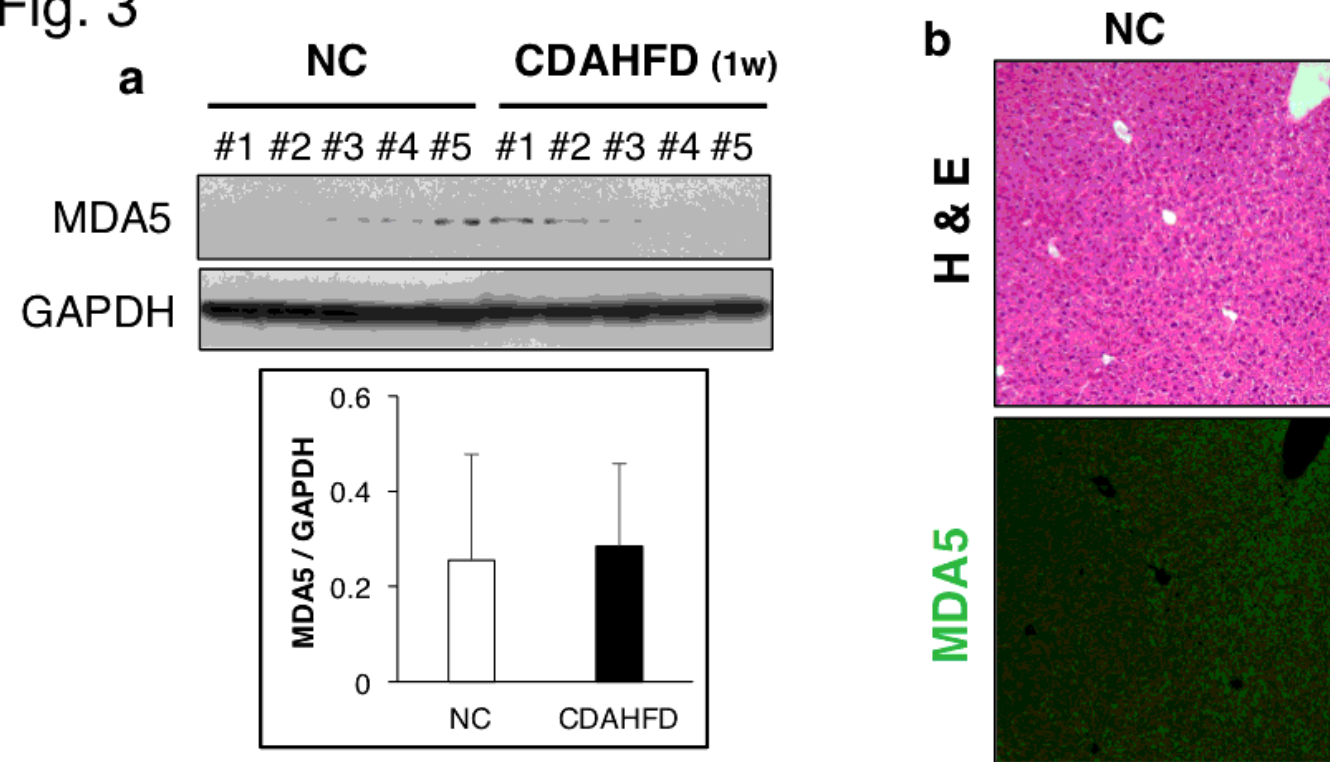

CDAHFD (1w)
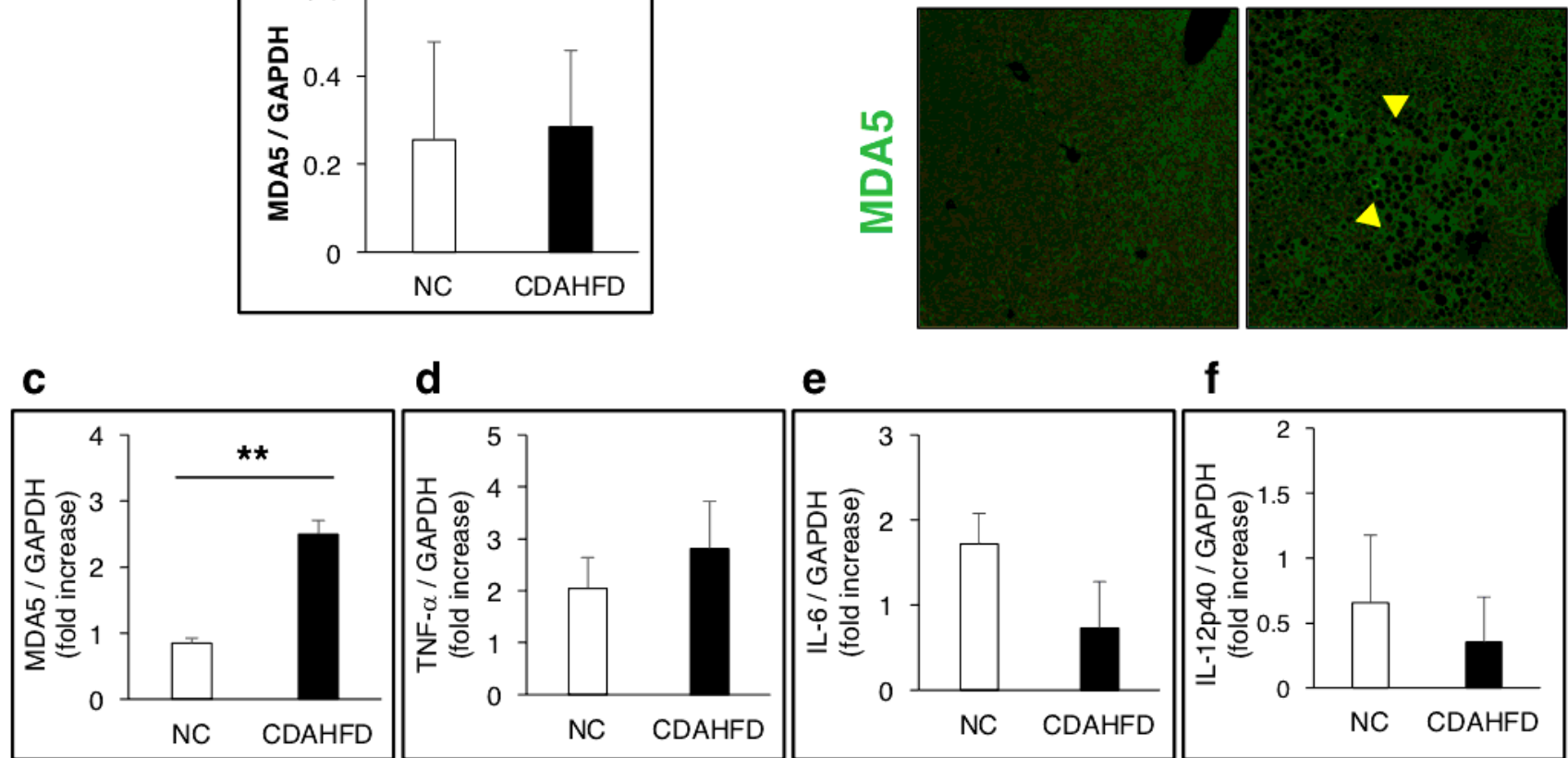

\section{Figure 3}

(a) MDA5 protein level remained unchanged at 1 week of CDAHFD. Results are presented as mean \pm SEM of five mice in each group. (b) Hematoxylin and eosin (H\&E) staining and immunohistochemical staining for MDA5 in the liver sections of NC and CDAHFD mice. MDA5-positive cells began to appear in CDAHFD mice at 1 week (yellow arrowhead). Original magnification: x100. mRNA expression of MDA5 (c), TNF-a (d), IL-6 (e), and IL12p40 (f) was analyzed by qPCR. MDA5 mRNA was significantly upregulated in the liver of CDAHFD mice. Results are presented as mean \pm SEM of three mice in each group. ${ }^{\star \star} p<0.01 \mathrm{vs}$. NC. 
Fig. 4
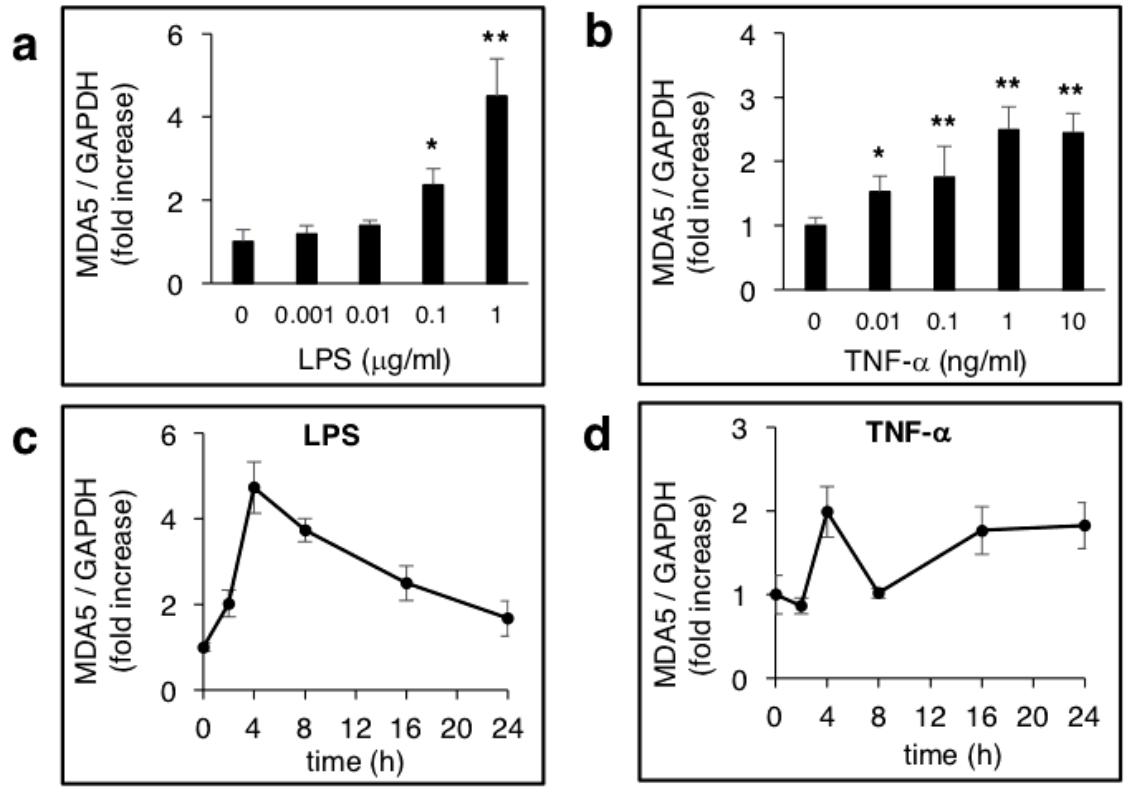

d
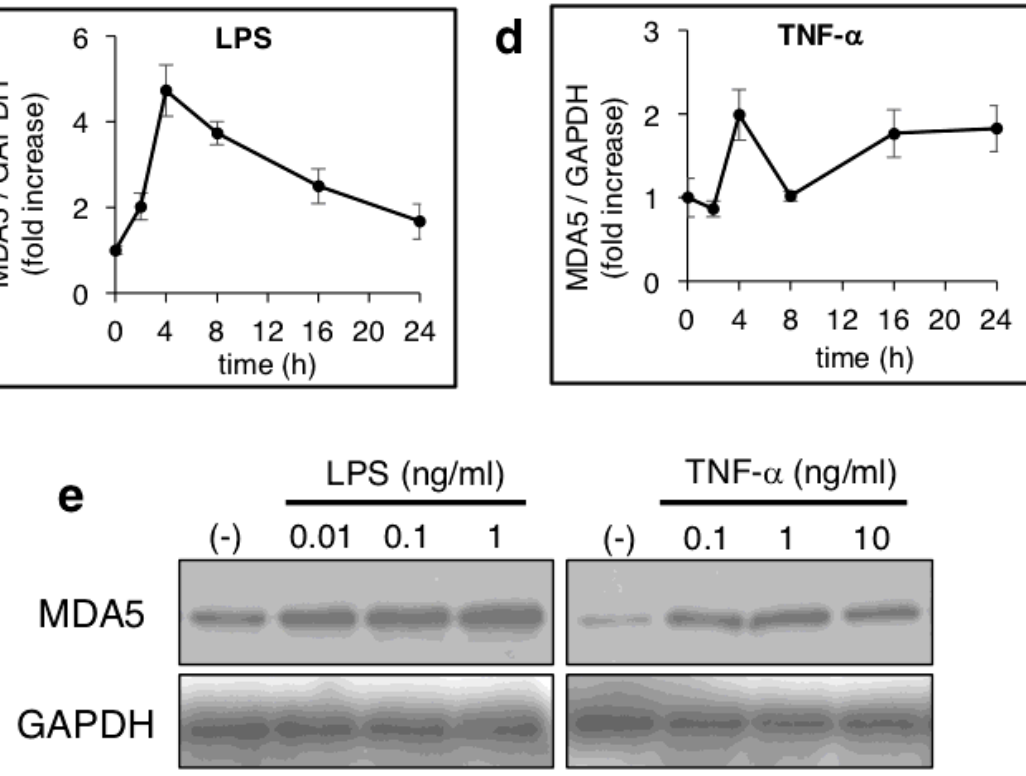

\section{Figure 4}

Stimulation with LPS or TNF-a induced the expression of MDA5 in THP-1 cells. PMA-differentiated THP-1 cells were stimulated with various concentration of LPS (a), or TNF-a (b) for $4 \mathrm{~h}$, and total RNA was extracted from the cells. Expression of MDA5 mRNA was analyzed by qPCR. Cells were treated with 1 $\mu \mathrm{g} / \mathrm{mL}$ of LPS (c), or $10 \mathrm{ng} / \mathrm{mL}$ of TNF-a (d) for upto $24 \mathrm{~h}$, and the MDA5 mRNA expression was analyzed by qPCR. Cells were incubated with various concentration of LPS or TNF-a for $24 \mathrm{~h}$, and the cell lysates were subjected to western blotting for MDA5 and GAPDH (e). 
Fig. 5

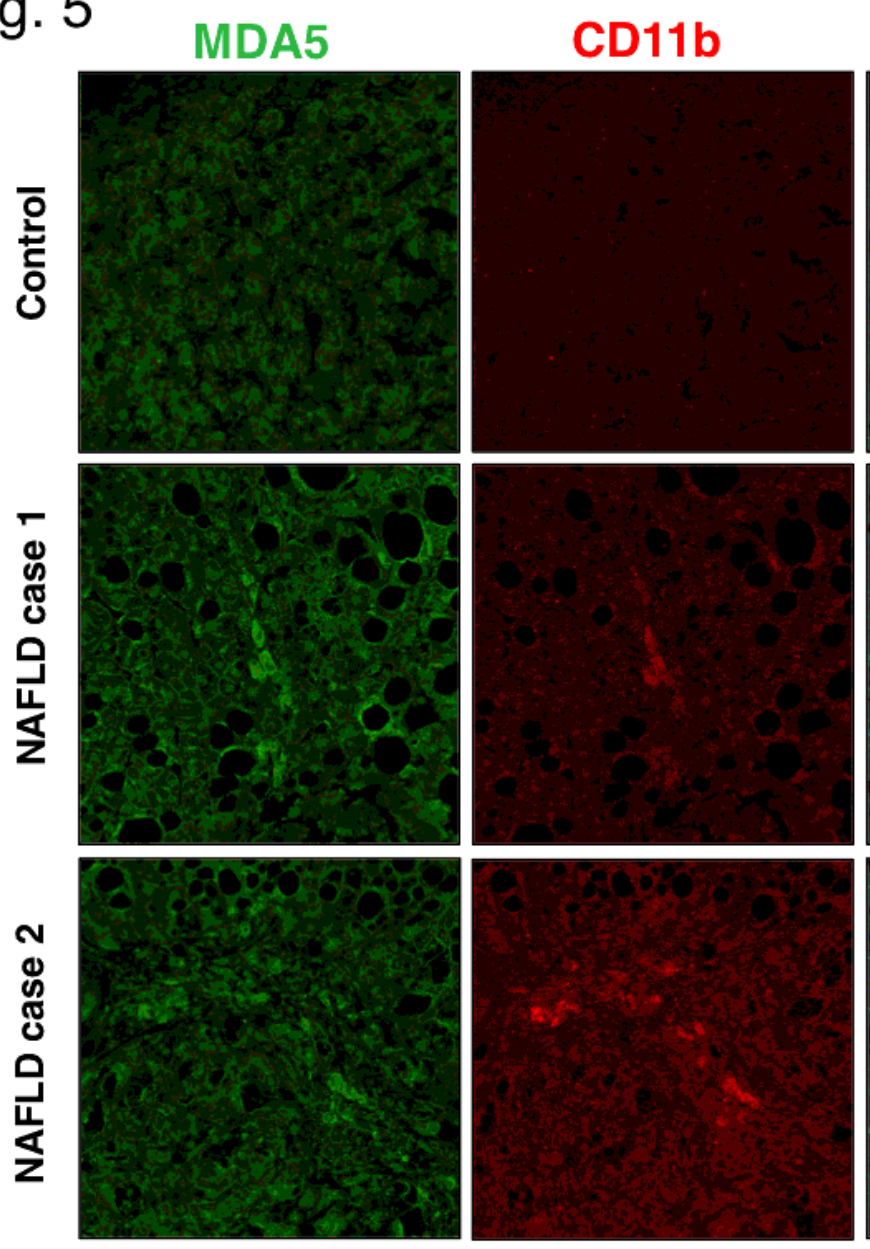

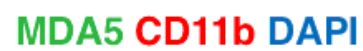
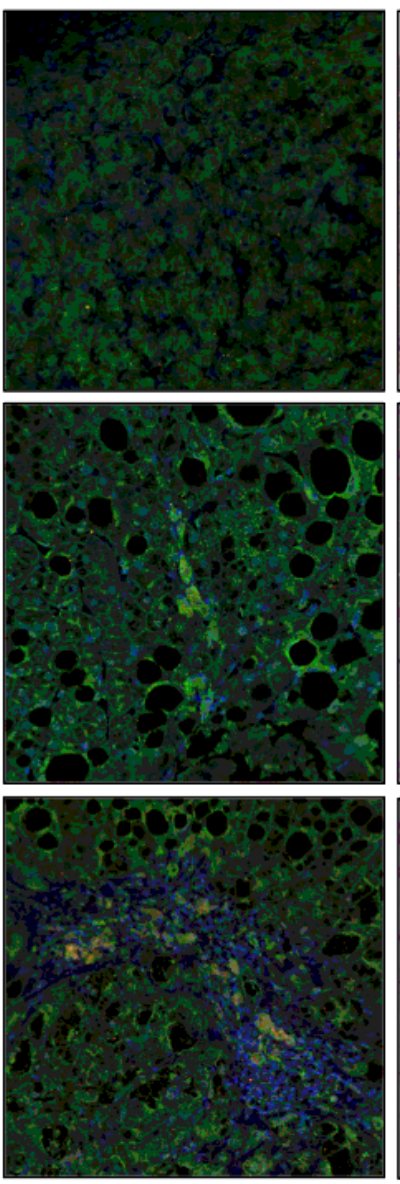

$H \& E$

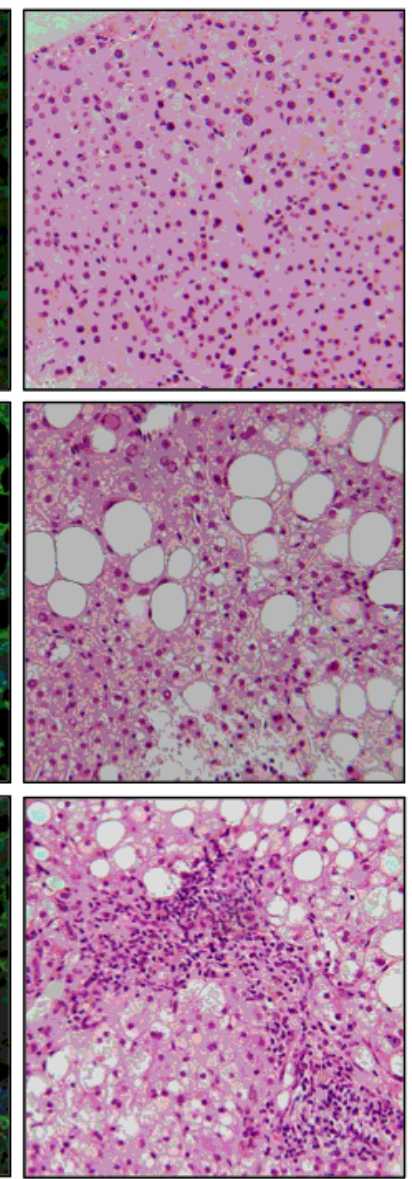

\section{Figure 5}

MDA5 was expressed in CD11b+ macrophages in human NAFLD. Liver biopsy specimens were obtained from eight patients with NAFLD and two cases without any existing liver diseases (control), and the sections were subjected to IHC and H\&E staining. Immunostaining was performed using anti-MDA5 (green) and anti-CD11b (red) antibodies. Cell nuclei were stained with DAPI (blue). Representative images of two cases with NAFLD and a control are shown. Original magnification: x200. 
Fig. 6

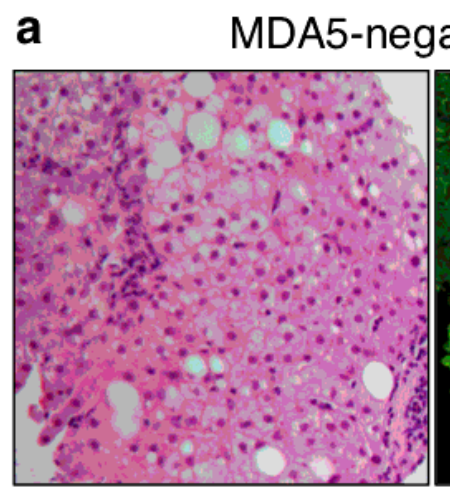

H \& E ative case

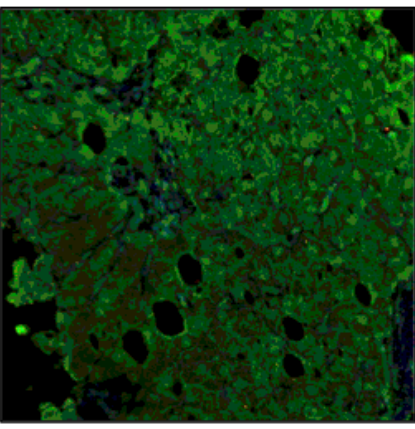

MDA5 CD11b DAPI

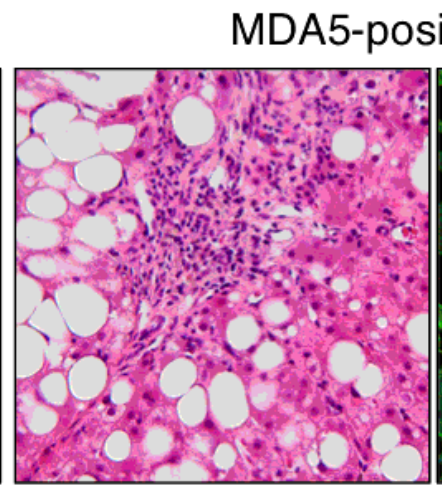

$H \& E$

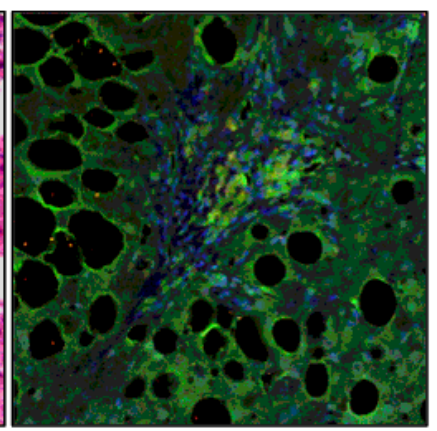

MDA5 CD11b DAPI
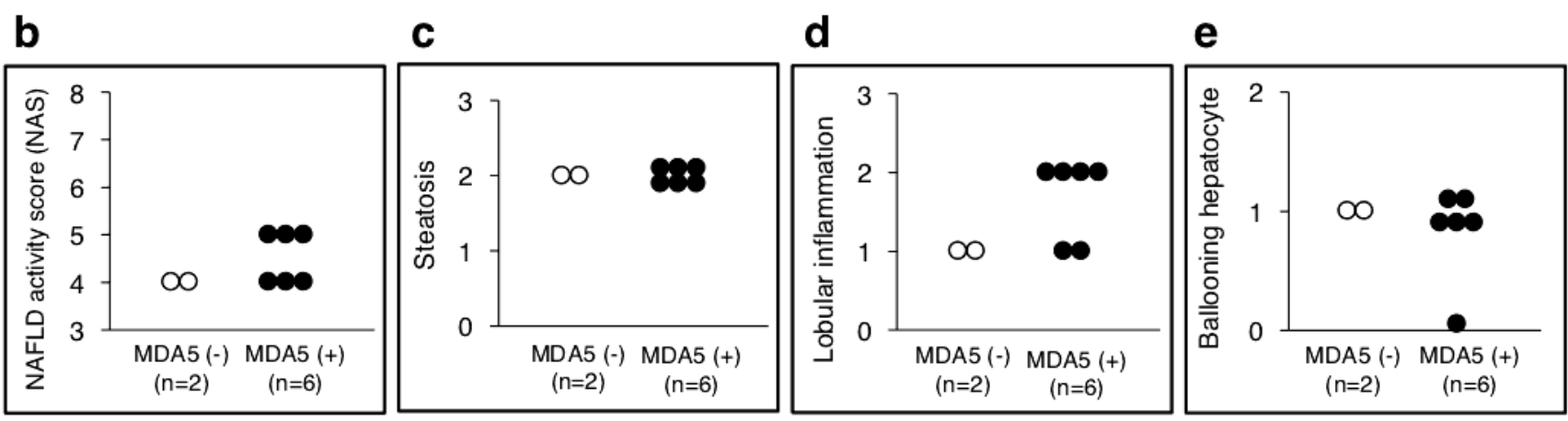

\section{Figure 6}

Eight NAFLD cases were classified based on MDA5 expression. As MDA5 expression was very low in two cases, they were regarded as MDA5-negative cases. Representative images of MDA5-positive cases and MDA5-negative cases are shown (a). Original magnification: x200. Liver biopsy specimens were subjected to H\&E staining to evaluate NAFLD activity score (NAS). NAS (b), steatosis (c), lobular inflammation (d), and ballooning (e) scores are presented in each group.

\section{Supplementary Files}

This is a list of supplementary files associated with this preprint. Click to download.

- Table1.tiff

- SupplFig.pdf

- Supplementary.pdf 